\title{
COMUNICAÇÃO
}

\section{AVALIAÇÃO DE ÓlEOS ESSENCIAIS DE CONDIMENTOS SOBRE O DESENVOLVIMENTO MICELIAL DE FUNGOS ASSOCIADOS A PRODUTOS DE PANIFICAÇÃO}

\author{
Evaluation of condiments essential oils on micelial \\ growth of fungi associated to bread-making products
}

\author{
Sára Maria Chalfoun de Souza ${ }^{1}$, Marcelo Cláudio Pereira ${ }^{2}$, Caroline Lima Angélico ${ }^{3}$, Carlos José Pimenta ${ }^{4}$
}

\section{RESUMO}

Este trabalho foi realizado com o objetivo de avaliar o efeito "in vitro" de óleos essenciais dos condimentos alho (Allium sativum L.), canela (Cinnamomum burnannil Meissn), cravo-da-índia (Caryophillus aromaticus L.) e tomilho (Thymus vulgaris L.), que foram adicionados aos meios de cultura (BDA E CYA20S). Os óleos foram extraídos pela técnica de arraste a vapor e testados nas concentrações de 500;1000; 1500 e $2000 \mu \mathrm{g} / \mathrm{mL}$, exceto o cravo, que foi testado nas concentrações de 200; 400; 600 e $800 \mu \mathrm{g} / \mathrm{mL}$. Como culturas-teste, foram utilizados os fungos Rhizopus sp.; Penicillium spp.; Eurotium repens e Aspergillus niger, isolados de pães envelhecidos. O óleo essencial da canela inibiu completamente o desenvolvimento dos fungos testados. Os óleos de tomilho e alho tiveram o mesmo efeito nas concentrações mais altas. O cravo inibiu o desenvolvimento dos fungos Aspergillus niger, Rhizopus sp. e Eurotium repens com a concentração de $600 \mu \mathrm{g} / \mathrm{mL}$ e do fungo Penicillium spp. com a de $800 \mu \mathrm{g} / \mathrm{mL}$.

Termos para indexação: Condimentos, óleos essenciais, fungos, inibição.

\section{ABSTRACT}

The objective of this work was to evaluate the "in vitro" effect of essential oils of garlic (Allium sativum L.), cinnamon (Cinnmomum burnannil Meissn), clove (Caryophillus aromaticus L.) and thyme (Thymus vulgaris L.) condiments that were added to culture media (PDA and CYA 20S). The essential oils were extracted by utilizing the vapor "dragging" technique and tested in the concentrations of 500;1000; 1500 and $2000 \mu \mathrm{g} / \mathrm{mL}$, exept clove, which was tested in concentrations of 200, 400, 600 and $800 \mu \mathrm{g} / \mathrm{mL}$. The fungi Rhizopus sp., Penicillium spp., Eurotium repens and Aspergillus niger, isolated of breads discarded for consumption. The cinnamon essential oil inhibited completely the tested fungi development. The thyme and garlic oils presented a pronounced effect at the higher concentrations. The essencial oil of clove inhibited the growth of Aspergillus niger, Rhizopus sp. and Eurotium repens at concetrations above $600 \mu \mathrm{g} / \mathrm{mL}$ and of Penicillium above $800 \mu \mathrm{g} / \mathrm{mL}$.

Index terms: Condiments; essential oils; fungi, inhibition.

(Recebido para publicação em 24 de setembro de 2003 e aprovado em 5 de janeiro de 2004)

O valor condimentar de uma planta está quase sempre associado ao teor de óleos essenciais, que são compostos químicos gerados durante o desenvolvimento da planta (FURLAN, 1998).

Por definição, condimentos e especiarias são produtos aromáticos de origem vegetal empregados principalmente para conferir sabor aos alimentos. Se- gundo Shelef (1983), além dessa utilidade, os condimentos possuem também propriedades antimicrobianas, antioxidantes e medicinais.

As propriedades antimicrobianas dos condimentos e de seus óleos essenciais têm sido estudadas principalmente com relação ao efeito inibidor de microrganismos patogênicos presentes em alimentos.

\footnotetext{
1. Engenheira Agrônoma, Dra., Pesquisadora da Empresa de Pesquisa Agropecuária de Minas Gerais/EPAMIG, Caixa Postal 176, 37200-000 Lavras, MG. chalfoun@ufla.br

2. Biólogo, M.Sc em Ciência dos Alimentos, área de concentração Microbiologia de Alimentos/EPAMIG, marcelo.claudio@posgrad.ufla.br 3. Engenheira Agrônoma, bolsista da EPAMIG, caroline.angelico@ig.com.br

4. Engenheiro Agrônomo, DS em Ciência dos Alimentos, Professor do Departamento de Ciência dos Alimentos da Universidade Federal de Lavras/UFLA - Caixa Postal 37-37200-000 - Lavras, MG. Carlos_pimenta@ufla.br
} 
Em vários estudos, tem sido evidenciado que os princípios ativos dos condimentos localizam-se na fração de óleo essencial (PARRY, 1962; HITOKOTO et al., 1980; PRUTHI, 1980; FARAG et al., 1989; KOKETSU e GONÇALVES, 1991; PATKAR et al., 1993a; MAHMOUD, 1994; FURLAN, 1998). Os óleos essenciais das espécies de condimentos contêm diferentes compostos, que contribuem com as propriedades antimicrobianas (PARRY, 1962; PRUTHI, 1980).

Óleos essenciais de canela a $200 \mu \mathrm{g} / \mathrm{mL}$ e de cravo a $250 \mu \mathrm{g} / \mathrm{mL}$ foram inibidores do desenvolvimento e da produção de toxina de Aspergillus parasiticus, ao passo que o aldeído cinâmico e o eugenol, principais constituintes desses óleos essenciais, apresentaram efeito inibidor a 150 e $125 \mu \mathrm{g} / \mathrm{mL}$, respectivamente, segundo Bullerman et al. (1977).

Em estudos envolvendo 32 óleos essenciais extraídos de condimentos, demonstrou-se que os óleos de pimenta-da-jamaica, canela, cravo, cebola, alho, orégano, segurelha e tomilho foram, em ordem decrescente, os maiores inibidores de oito gêneros de levedura. (CONNER et al., 1984a, b).

$\mathrm{O}$ efeito dos óleos de alho e de cebola foi estudado na produção da toxina botulínica, verificando-se que na concentração $1.500 \mu \mathrm{g} / \mathrm{mL}$ ambos inibiram a produção da toxina tipo $\mathrm{A}$, mas não afetaram a produção das toxinas tipo B e E (DEWIT et al., 1979).

Garc e Siddiqui (1992) realizaram trabalhos com substâncias isoladas do óleo essencial de manjericão (Ocimum sanctum) e evidenciaram ação fungistática em diversos fungos, o eugenol purificado foi testado na diluição de 1:100 e 1:200, apresentando forte ação contra Absidia glauca, Alternaria alternata, Aspergillus niger, Colletotrichum capsici, Fusarium moniliforme e Rhizopus nodosus. Singh et al. (1993) utilizaram extrato aquoso de manjericão em frutas de banana para controle de doenças provocadas por fungos dos gêneros Fusarium, Helminthosporium, Curvularia, Aspergillus e Trichothecium e obtiveram resultados eficientes.

Ao avaliar o efeito do timol, principal componente dos óleos de orégano e de tomilho, Buchanan e Shepard (1981) constataram uma atividade inibidora da síntese de aflatoxinas significante, decorrente da inibição do desenvolvimento fúngico.

O óleo essencial de menta e o mentol podem ser usados na composição de muitos produtos, pois seus efeitos sobre os microrganismos podem ser aproveita- dos de várias maneiras (MAIA, 1994). Singh et al. (1993) demonstraram o efeito fungicida e fungistático desse óleo sobre 23 espécies, entre elas Alternaria sp, Curvalaria lunata, Fusarium moniliforme, $F$. solani e Rhizoctonia bataticola; os autores usaram concentrações que variam de 500 a $10.000 \mu \mathrm{g} / \mathrm{mL}$ de óleo de menta nos respectivos meios de cultura, e observaram inibição de $100 \%$ do crescimento micelial, a partir de $2.000 \mu \mathrm{g} / \mathrm{mL}$.

Com o presente trabalho, teve-se como objetivo determinar o efeito da adição de óleos essenciais em meios específicos no desenvolvimento micelial dos fungos Rhizopus sp.; Penicillium spp.; Eurotium repens e Aspergillus niger, e foi desenvolvido no Laboratório de Fitopatologia do EcoCentro/ EPAMIG, localizado na Universidade Federal de Lavras, MG.

Os organismos testados foram obtidos de pães descartados para o consumo por apresentarem sinais de contaminação e constam da micoteca do Laboratório de Fitopatologia da EPAMIG.

Os óleos essenciais dos condimentos canela, alho e tomilho, comprovadamente isentos de contaminação mediante testes "in vitro", foram acrescentados nas concentrações de 500,1000,1500 e 2000 $\mu \mathrm{g} / \mathrm{mL}$ aos meios de cultura BDA (Batata-DextroseAgar) para os fungos Aspergillus niger e Rhizopus sp. e CYA+20S (Czapek Yeast Extract Agar + 20\% Sucurose) para os fungos Eurotim repens e Penicillium spp., e o cravo foi testado em concentrações de $200,400,600$ e $800 \mu \mathrm{g} / \mathrm{mL}$.

Após sete dias de incubação em BOD (Mod. 347 CDG) com temperatura de $25^{\circ} \mathrm{C}$ e fotoperíodo de 12 horas, foram efetuadas medições ortogonais do diâmetro das colônias, tendo como referência o desenvolvimento da placa-controle.

Para obtenção dos óleos essenciais das plantas, utilizou-se a metodologia de arraste a vapor descrita por Koketsu e Gonçalves (1991).

Foram pesados $50 \mathrm{~g}$ do condimento em pó (cravo) e $100 \mathrm{~g}$ dos demais condimentos, em folhas secas (tomilho), casca (canela) e bulbos (alho), ficando esses em contato direto com 1 litro de água em ebulição, flutuando ou imerso no líquido, dependendo da densidade ou da quantidade de material colocado no recipiente de destilação. A água foi posta em ebulição por aquecimento direto, conduzindo o vapor d'água.

Os resultados dos tratamentos com óleos essenciais sobre o desenvolvimento micelial dos fungos estu-

Ciênc. agrotec., Lavras, v. 28, n. 3, p. 685-690, maio/jun., 2004 
dados encontram-se representados nas Figuras 1, 2, 3 e 4. Observa-se que os óleos essenciais obtidos dos condimentos apresentaram elevado índice de inibição do desenvolvimento micelial dos fungos estudados, quando comparados com a testemunha não tratada.

Com relação ao cravo, na Figura 1, as concentrações testadas foram mais reduzidas que os dos demais condimentos, baseando-se na elevada eficiência apresentada pelo condimento quando testado na forma de pó em ensaios anteriores (BULLERMAN et al., 1977, HITOKOTO et al., 1980; BARA, 1992). Observa-se que a partir de 600 $\mu \mathrm{g} / \mathrm{mL}$, todos os fungos estudados tiveram o desenvolvimento micelial inibido, com exceção do Penicillium spp., embora também tenha apresentado uma acentuada inibição do desenvolvimento quando comparado à testemunha (sem adição de óleo). Os resultados são concordantes com aqueles obtidos anteriormente (HITOKOTO et al., 1980; AZZOUS et al., 1982; SHELEF, 1983), nos quais se evidencia o elevado poder antimicrobiano do cravo.

Pelos resultados referentes à adição do óleo essencial de alho, conforme representado na Figura 2, demonstra-se que ele controlou o desenvolvimento mi- celial de todos os fungos, exceto o fungo Rhizopus sp., que foi controlado apenas na maior concentração testada. Com tais resultados, confirmam-se estudos anteriores, os quais têm comprovado as propriedades antimicrobianas e medicinais desse condimento (CAVALLITO e BAYLEY, 1944; CAVALLITO et al., 1945, CONNER et al., 1984a, b).

Na Figura 3 pode-se observar que o óleo essencial de canela apresentou um controle total (100\%) do desenvolvimento micelial de todos os fungos testados a partir da concentração de 500 $\mu \mathrm{g} / \mathrm{mL}$, confirmando dados anteriores sobre a eficiência desse condimento, quando adicionado sob a forma de pó e óleo essencial (BULLERMAN et al., 1977; TIWARI et al., 1983; PATKAR et al., 1993b; SINHA et al., 1993).

Com relação ao tratamento de adição de óleo essencial de tomilho ao meio (Figura 4), observa-se que o óleo inibiu o crescimento micelial dos fungos testados em todas as concentrações, resultados esses concordantes com os obtidos pela adição desse condimento em pó e por pesquisas anteriores, por meio das quais revelouse o seu poder antimicrobiano (MARUZZELLA e LIGUORI, 1958; BEUCHAT, 1976; FARAG et al., 1989).

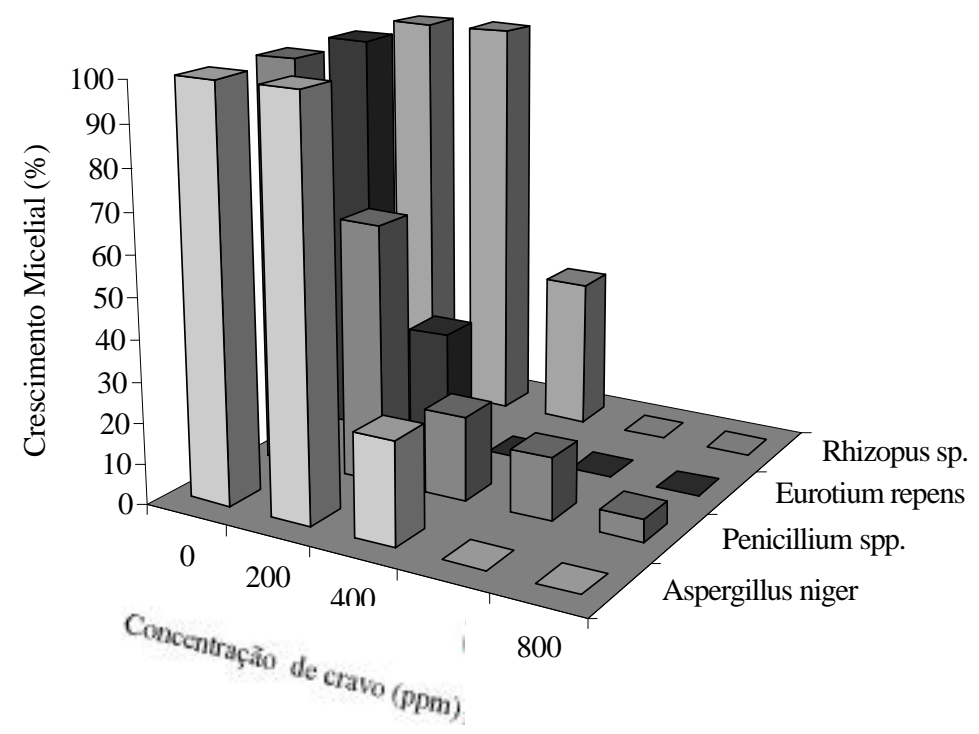

FIGURA 1 - Efeito do óleo essencial de cravo sobre o desenvolvimento micelial dos fungos associados aos produtos de panificação, após sete dias de incubação nos meios de cultura BDA e CYA+20S. 


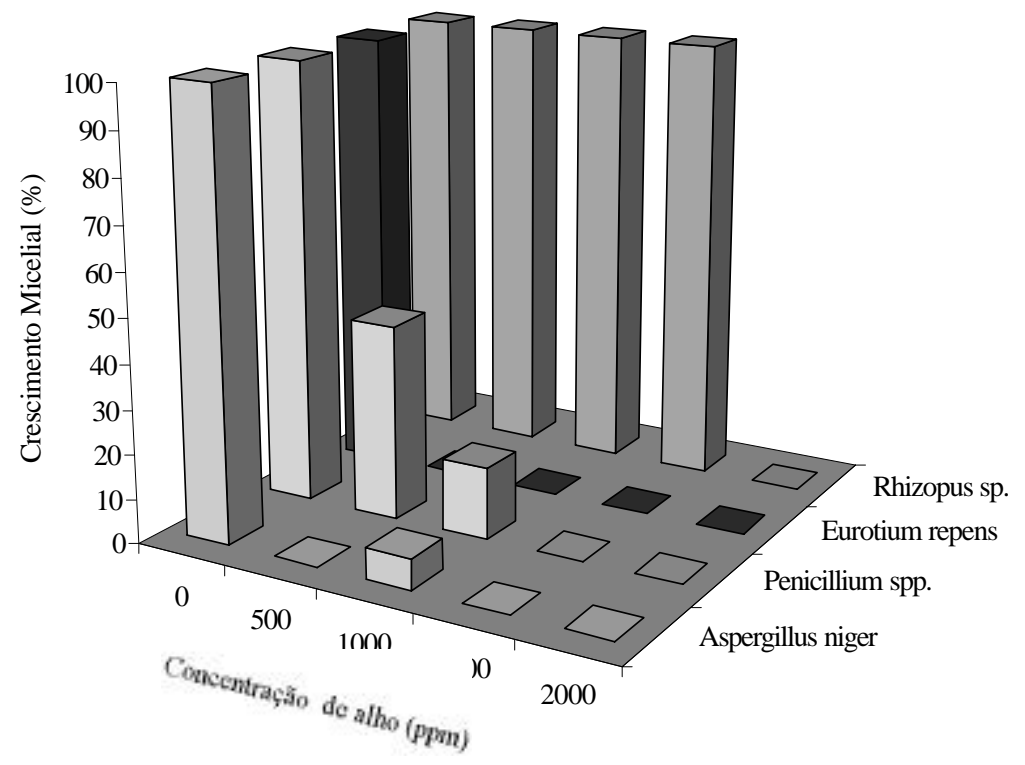

FIGURA 2 - Efeito do óleo essencial de alho sobre o desenvolvimento micelial dos fungos associados aos produtos de panificação, após sete dias de incubação nos meios de cultura BDA e CYA+20S.

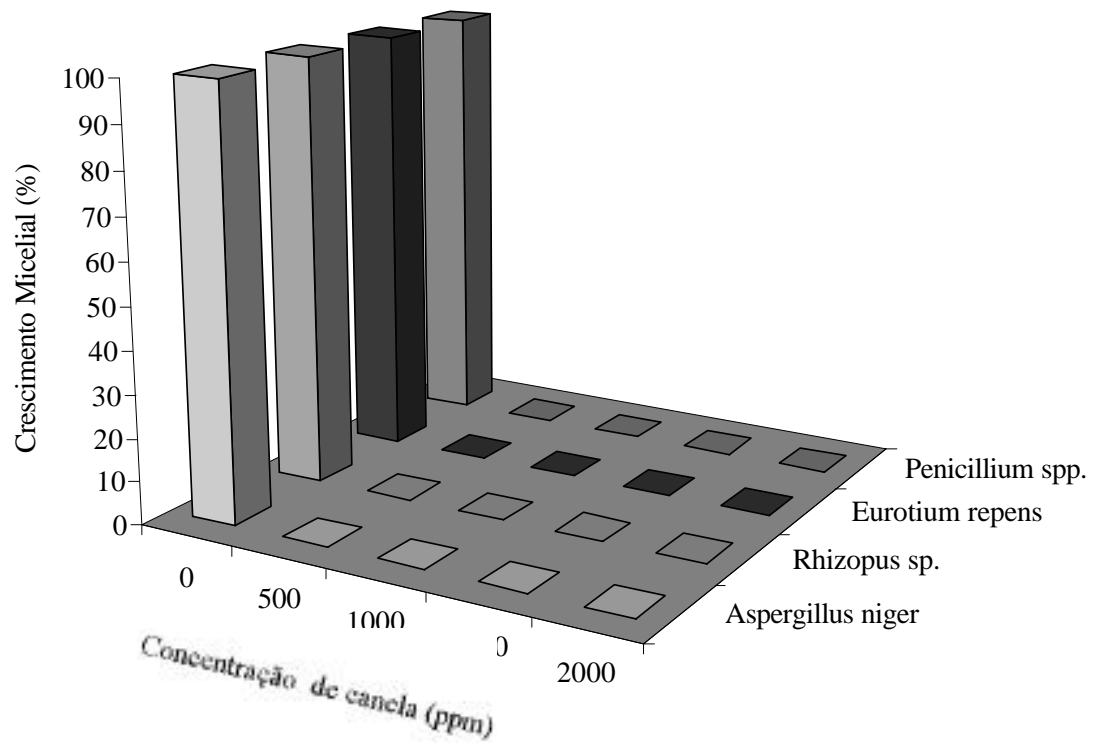

FIGURA 3 - Efeito do óleo essencial de canela sobre o desenvolvimento micelial dos fungos associados aos produtos de panificação, após sete dias de incubação nos meios de cultura BDA e CYA+20S. 


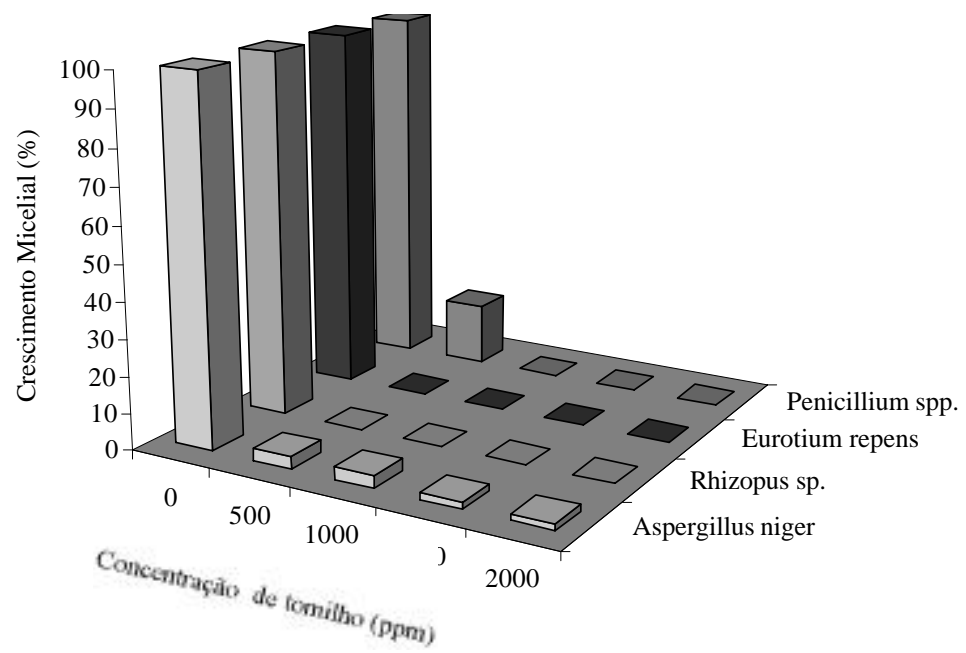

FIGURA 4 - Efeito do óleo essencial de tomilho sobre o desenvolvimento micelial dos fungos associados aos produtos de panificação, após sete dias de incubação nos meios de cultura BDA e CYA+20S.

A eficiência apresentada pelos condimentos adicionados sob a forma de óleos essenciais do cravo, canela, tomilho e alho sobre a inibição do desenvolvimento micelial de fungos associados a produtos de panificação confirmam o fato de que grande parte dos princípios ativos desses condimentos encontra-se concentrada nessa fração (PARRY, 1962; HITOKOTO et al., 1980; PRUTHI, 1980; FARAG et al., 1989; KOKETSU e GONÇALVES, 1991). Os condimentos adicionados a meios específicos sob a forma de óleos essenciais do cravo, canela, tomilho e alho inibiram o desenvolvimento micelial dos fungos, confirmando a sua eficiência fungicida e/ou fungistática.

\section{REFERÊNCIAS BIBLIOGRÁFICAS}

AZZOUS, M. A.; BULLERMAN, L. R. Comparative antimycotic effects of selected herbs, spices, planta components and commercial anti-fungal agents. Journal of Food Protection, Ames, v. 45, n. 14, p. 1298-1301, Dec. 1982.

BARA, M. T. F. Avaliação do efeito inibidor de condimentos no desenvolvimento de Yersinia enterocolitica. 1992. 73 f. Dissertação (Mestrado em Microbiologia Agrícola) - Universidade Federal de Viçosa, Viçosa, 1992.

BEUCHAT, L. R. Sensitivity of vibrio parahemolyticus to spices and organic acids. Journal of Food Science, Chicago, v. 41, n. 4, p. 899-902, July/Aug. 1976.
BUCHANAN, R. L.; SHEPARD, A. J. Inhibition of Aspergillus parasiticus by timol. Journal of Food Science, Chicago, v. 46, p. 976-977, 1981.

BULLERMAN, L. B.; LIEW, F. Y.; SEIER S. A. Inhibition of growth and aflatoxin production by cinamon and clove oils, cinnamic aldehyde and eugenol. Journal of Food Science, Chicago, v. 42, n. 6, p. 1107-1109, Nov./Dec. 1977.

CAVAllito, J. C.; BAYLEY, J. H. Allicin, the antibacterial principle of Allium sativum: (i) isolation, physical properties and antibacterial action. Journal of the American Chemical Society, Easton, v. 66, p. 1950-1951, 1944.

CAVAllito, J. C.; BAYLEY, J. H.; BUCK, J. S. The anti-bacterial principle of Allium sativum: 3. its precursor and "essential oil of garlic." Journal of the American Chemical Society, Easton, v. 67, p. 1032, 1945.

CONNER, D. E.; BEUCHAT, L. R. Effects of essential oils from plants on growth of food spoilage yeasts. Journal Food Science, Chicago, v. 49, p. 429-434, 1984a.

CONNER, D. E.; BEUCHAT, L. R. Sensitivity of heatstressed yeasts to essential oils of plants. Applied Environmental Microbiology, Washington, v. 47, n. 2, p. 229-233, Feb. 1984b. 
DEWIT, J. C.; NOTERMANS, S.; GORIN, N.; KAMPELMACHER, E. H. Effects of garlic and onion oil on toxin production by $\mathrm{C}$ : botulinum in meat slurry. Journal of Food Protection, Ames, v. 42, p. 222-224, 1979.

FARAG, R. S.; DAW, Z. Y.; ABO-RAYA, S. H. Influence of some spice essencial oils on Aspergillus parasiticus growth and production of aflatoxinas in a synthetic medium. Journal of Food Science, Chicago, v. 54, n. 1, p. 54-74, Jan./Feb. 1989.

FURLAN, M. R. Ervas e temperos: cultivo e comercialização. Cuiabá: SEBRAE/MT, 1998. v. 15, 128 p.

GARC, S. C.; SIDDIQUI, N. Antifungal activity of some essential oil isolates. Phormazil., [S.1.], v. 47, n. 6, p. 467-468, 1992.

HITOKOTO, H.; MOROZUMI, S.; WAUKE, T.; SAKAI, S.; KURATA, H. Inhibitory effects of spices on growth and toxin production of toxigenic fungi. Applied and Evironmental Microbiology, Washington, v. 39, n. 4, p. 818-822, Apr. 1980.

KOKETSU, M.; GONÇALVES, S. L. Óleos essenciais e sua extração por arraste a vapor. Rio de Janeiro: EMBRAPA-CTAA, 1991. 24 p. (Documentos, 8).

MAHMOUD, L. E. Antifungal action and antiaflatoxigenic properties of some essential oil constituents. Letters in Applied Microbiology, Oxford, n. 2, p. 110-114, 1994.

MAIA, N. B. Nutrição mineral, desenvolvimento e qualidade do óleo essencial da menta (Mentha arvensis L.) cultivada em solução nutritiva. 1994. 69 f. Dissertação (Mestrado) - Escola Superior de Agricultura de Luiz de Queiroz, Piracicaba, 1994.

MARUZZELLA, J. C.; LIGUORI, L. The in vitro antifungal activity of essential Oliz. Journal of the
American Pharmaceutical Association, Washington, v. 47 , p. $250-258,1958$.

PARRY, J. W. Spices: morphology, histology, chemistry. New York: Chemical, 1962. v. 2, 183 p.

PATKAR, K. L.; USHA, C. M.; SHETTY, H. S.; PASTER, N.; LACEY, J. Effect of spice oils on growth and aflatoxin $\mathrm{B}_{1}$ production by Aspergillus flavus. Letters in Applied Microbiology, Oxford, v. 17, p. 49-51, 1993a.

PATKAR, K. L.; USHA, C. M.; SHETTY, H. S.; PASTER, N.; LACEY, J. Effect of spice essential oils on growth and aflatoxin $B 1$ produciton by Aspergillus flavus. Letters in Applied Microbiology, Oxford, v. 17, n. 2, p. 49-51, Aug. 1993b.

PRUTHI, J. S. Spices and condiments: chemistry, microbiology, techonology. New York: Academic, 1980. 449 p.

SHELEF, L. A. Antimicrobial effects os spices. Journal of Food Safety, Connecticut, v. 6, n. 1, p. 2944, Aug. 1983.

SINGH, H. N. P.; PRASAD, M. M.; SINHA, K. K. Efficacy of leaf extracts of some medicinal plantas against disease deve lop ment in banana. Letters in Applied Microbiology, Oxford, v. 17, n. 6, p. 269-271, Dec. 1993.

SINHA, K. K.; SINHA, A. K.; GAJENDRA, P. The effect of clove and cinnamon oils on growth of and aflatoxin production by Aspergillus flavus. Letters in Applied Microbiology, Oxford, v. 16, p. 114-117, 1993.

TIWARI, R.; DIKSHIT, R. P.; CHANDAN, N. Inhibition of growth and aflatoxin $\mathrm{B}_{1}$ production of Aspergillus parasiticus by spice. Journal of Food Science and Tecnology, India, v. 20, p. 131-132, 1983. 